Funding The Netherlands Organisation for Health Research and Development (ZonMw).

\section{OP31 DEVELOPING AND IMPROVING A WEB-BASED TOOL FOR CLIENTS IN LONG-TERM CARE: A USER-CENTRED DESIGN}

C van Leersu*, A Moser, B van Steenkiste, E Stoffers, M Reinartz, J Wolf, T van der Weijden. Maastricht University, Maastricht, Netherlands

\subsection{6/spcare-2019-ACPICONGRESSABS.31}

Background The decision-making process for clients in need of long-term care is challenging and clients need to make choices about the care they prefer. A tool to assist the clients and caregivers with the decision-making and elicitation on preferences could be beneficial. The aim is to investigate userrequirements of a tool for the decision-making.

Methods We applied a user-centred design to develop this tool. This was an interactive process of collecting data with end-users and improving the prototypes. The end-users included clients, relatives, and caregivers. Four end-users participated in a development team and 22 end-users were interviewed individually. We collected data during three phases of iteration: look and feel, navigation, and content. We analysed the data using thematic analysis and adjusted the prototype after each phase.

Results The lay-out was approved by all participants during the look and feel phase, but there was a need for different/ neutral pictures. During the navigation phase, participants experienced easy navigation, but text-blocks had to be shortened. Considering the content, participants missed questions about well-being/happiness. After the third phase, the tool was finalized.

Conclusion The user-centred design was necessary to move from the prototypes to the finalized tool fitting usabilityrequirements of end-users. The tool 'What matters to me' (http://www.watikbelangrijkvind.nl) is currently in the feasibility-testing phase.

\section{OP32 USING TWITTER TO EXPLORE ADVANCE CARE PLANNING AMONG BRAIN TUMOR STAKEHOLDERS}

${ }^{1} \mathrm{H}$ Lum*, ${ }^{2} \mathrm{~N}$ Cutshall, ${ }^{4} \mathrm{~L}$ Salmi, R Berry, ${ }^{3} \mathrm{~B}$ Kwan. ${ }^{1}$ University of Colorado and VA GRECC, Aurora, USA; ${ }^{2}$ University of Colorado, Aurora, USA; ${ }^{3}$ university Of Colorado School of Medicine, Aurora, USA; ${ }^{4}$ Beth Israel Deaconess Medical Center, Boston, USA

\subsection{6/spcare-2019-ACPICONGRESSABS.32}

Background Advance care planning (ACP) often occurs too late in the disease course of patients affected by brain tumors. Furthermore, the perspectives of brain tumor stakeholders on ACP are not well described. This study uses a social media tweet chat to understand perspectives on ACP among brain tumor stakeholders.

Methods This qualitative descriptive study analyzed a tweet chat (real-time virtual group discussion on the social media platform Twitter) of brain tumor stakeholders. The 1-hour tweet chat was organized by the patient-run Twitter community referred to with the hashtag \#BTSM, which stands for Brain Tumor Social Media. Participants reflected on four questions about ACP by including \#BTSM in tweets. Unique tweets and stakeholder type (i.e. patient, caregiver, advocate or organization member, clinical provider and researcher, leader) were categorized. The tweet chat transcript was analyzed to identify key themes.

Results Fifty-two participants from four countries contributed 336 unique Tweets. Most participants were patients, clinical providers or researchers, and advocates or organizations. There were four key themes regarding brain tumor stakeholder perspectives about ACP: 1) cultural barriers prevent discussions of death; 2) ensuring one's voice is heard; 3) Goldilocks' approach to timing - fearing ACP is too early or too late; and 4) crowdsourcing ACP resources.

Conclusions A multi-national group of brain tumor stakeholders engaged in ACP discussions via a social media tweet chat and highlighted important challenges and opportunities. Social media is a new avenue in which clinicians and patients may engage with to better understand each other's perspectives related to ACP.

\section{OP33 NATIONWIDE MULTICENTER EVALUATION TO DETERMINE WHETHER PATIENT VIDEO TESTIMONIALS CAN SAFELY HELP ENSURE APPROPRIATE CRITICAL VERSUS END-OF-LIFE CARE}

${ }^{1} \mathrm{~F}$ Mirarchi, ${ }^{2} \mathrm{M}$ Markley*. Institute on Healthcare Directives, Erie, PA, USA; ${ }^{2} \mathrm{M}$ Jane Markley Consulting, LLC, Derwood, MD, USA

\subsection{6/spcare-2019-ACPICONGRESSABS.33}

Background End-of-life interventions should be predicated on consensus understanding of patient wishes. Written documents are not always understood; adding a video testimonial/message (VM) might improve clarity. Study goals were to (1) determine baseline rates of consensus in assigning code status and resuscitation decisions in critically ill scenarios and (2) determine whether adding a VM increased consensus.

Methods We randomly assigned 2 web-based survey links to 1366 faculty and resident physicians at institutions with graduate medical education programs in emergency medicine, family practice, and internal medicine. Each survey asked for code status interpretation of stand-alone Physician Orders for Life-Sustaining Treatment (POLST) and living will (LW) documents in 9 scenarios. Respondents assigned code status and resuscitation decisions to each scenario. For 1 of 2 surveys, a VM was included to help clarify patient wishes.

Results Response rate was 54\%, and most were male emergency physicians who lacked formal advanced planning, document interpretation training. Consensus was not achievable for stand-alone POLST or LW documents (68\%-78\% noted "DNR"). Two of 9 scenarios attained consensus for code status (97\%-98\% responses) and treatment decisions (96\%- 99\%). Adding a VM significantly changed code status responses by $9 \%$ to $62 \%(\mathrm{P} \leq 0.026)$ in 7 of 9 scenarios with 4 achieving consensus. Resuscitation responses changed by $7 \%$ to $57 \%(\mathrm{P} \leq 0.005)$ with 4 of 9 achieving consensus with VMs.

Conclusions For most scenarios, consensus was not attained for code status and resuscitation decisions with stand-alone LW and POLST documents. Adding VMs produced significant impacts toward achieving interpretive consensus. 